\title{
Optimization of hybrid Raman-Brillouin-EDF amplification fiber laser in long distance FBG sensor system
}

\begin{abstract}
We present a hybrid Raman-Brillouin-Erbium Doped Fiber (EDF) amplification based Fiber Bragg Grating (FBG) sensor system capable of transmitting over long reach Single Mode Fiber (SMF). The performance of output spectra has been optimized and realized by the best effective placement of EDF and Raman pump power. An optimized combination of Raman, Brillouin and Erbium gain brings forth an optical signal-to-noise ratio of $16.34 \mathrm{~dB}$ in $100 \mathrm{~km}$ long distance FBG sensor system.
\end{abstract}

Keyword: Erbium doped fiber; FBG sensor; Fiber optic sensor; long distance sensor; Raman amplification 